\title{
Philonsorbonne
}

\section{Anatomie du sens moral : Hume et Hutcheson}

\section{Lisa BROUSSOIS}

\section{OpenEdition}

\section{Journals}

Édition électronique

URL : https://journals.openedition.org/philonsorbonne/478

DOI : 10.4000/philonsorbonne.478

ISSN : 2270-7336

\section{Éditeur}

Publications de la Sorbonne

\section{Édition imprimée}

Date de publication : 15 novembre 2013

Pagination : 45-62

ISSN : 1255-183X

\section{Référence électronique}

Lisa BROUSSOIS, «Anatomie du sens moral : Hume et Hutcheson», Philonsorbonne [En ligne], 7|

2013, mis en ligne le 18 décembre 2013, consulté le 08 juin 2021. URL : http://

journals.openedition.org/philonsorbonne/478; DOI : https://doi.org/10.4000/philonsorbonne.478

(c) Tous droits réservés 


\title{
Anatomie du sens moral : Hume et Hutcheson
}

\author{
Lisa Broussois
}

\section{Introduction}

En 1739, après la publication des deux premières parties de son Traité de la Nature Humaine, David Hume est fortement déçu par l'accueil des lecteurs. Il cherche alors le soutien d'un grand nom de la philosophie pour appuyer la parution de sa troisième partie sur la morale. Francis Hutcheson, professeur de philosophie morale à l'université de Glasgow et autorité incontestable de l'époque, s'engage dans une correspondance littéraire avec Hume et accepte d'envoyer ses remarques sur la partie non publiée du Traité, ce qu'il fait dès l'été ou l'automne 1739.

Dans une des lettres de Hume à Hutcheson, celle du 17 septembre 1739, Hume répond à une critique générale que lui fait Hutcheson : un manque d'enthousiasme pour défendre la cause de la vertu. L'auteur du Traité, pour se justifier, développe une comparaison assez explicite entre sa philosophie et celle de Hutcheson afin de montrer que les deux suivent une méthode relativement opposée, ce qui explique, selon lui, pourquoi il ne peut agir autrement :

Il y a différentes manières d'examiner l'esprit aussi bien que le corps. On peut les considérer en anatomiste ou en peintre; soit pour en découvrir les ressorts et les principes les plus secrets, soit pour décrire la grâce et la beauté de ses actions. J'imagine qu'il est impossible de combiner ces deux points de vue. $\mathrm{Si}$ vous dépouillez la peau et que vous mettez à jour les plus petites parties, quelque chose de trivial apparaît même dans les plus nobles attitudes et dans les actions les plus vigoureuses. Et vous ne pouvez jamais rendre l'objet gracieux ou engageant si vous n'habillez pas à nouveau les parties de peau et de chair et si vous les présentez seulement dans leur nudité ${ }^{\text {. }}$ 
La distinction que Hume fait entre l'anatomiste et le peintre se rencontre également dans le Traité de la nature humaine, en III iii 66, et dans l'introduction de l'Enquête sur l'entendement humain. En vérité, cette comparaison dépasse la simple considération de deux méthodes différentes. Sur ce point, les interprétations sont très variées. Il n'existe pas de consensus entre les commentateurs à ce sujet ${ }^{2}$. On a tantôt interprété la comparaison du peintre et de l'anatomiste comme deux styles d'écriture différents, tantôt comme deux genres littéraires, parfois même deux objectifs philosophiques complémentaires $^{3}$. On distingue également la méthode, la perspective et l'audience de destination ${ }^{4}$. Koorsgard montre que si l'un recherche les causes de l'approbation morale (l'anatomiste) et l'autre cherche à rendre la vertu séduisante (le peintre), aucun des deux ne s'occupe réellement de la fondation de la morale et des questions de justification des principes moraux ${ }^{5}$. Immerwahr et Abramson, quant à eux, défendent que Hume fut à la fois anatomiste et peintre : les deux représentant une forme de philosophie humienne ${ }^{6}$.

Loin d'entrer dans les détails de ces considérations, nous en resterons à une tentative d'application de cette distinction en privilégiant le point de

the mind as well as the body. One may consider it either as an anatomist or as a painter: either to discover its most secret springs and principles, or to describe the grace and beauty of its actions. I imagine it impossible to combine these two views. Where you pull off the skin and display all the minute parts, there appears something trivial, even in the noblest attitudes and most vigorous actions; nor can you ever render the object graceful or engaging, but by clothing the parts again with skin and flesh, and presenting only their bare outside. (Ma traduction en français).

2. Les commentateurs qui se sont penchés sur le sujet sont trop nombreux pour être énumérés ici. Nous ne pouvons qu'en indiquer quelques uns: Malherbe, Buckle, Stewart, Moore, Wood, Shaver, etc. (voir quelques références dans la bibliographie finale, p. 60).

3. Immerwahr propose une distinction entre deux justifications différentes (J. Immerwahr, " The Anatomist and the Painter: The Continuity of Hume's Treatise and Essays », Hume Studies XVII, 1 (April, 1991), p. 1-14). L'anatomie donne une assistance à la philosophie pratique (peinture) et le peintre aide les hommes à vivre une vie vertueuse et plus heureuse. Abramson affirme, cependant, que servir la peinture ne peut pas être le seul but de l'anatomie (K. Abramson, «Hume's Distinction between Philosophical Anatomy and Painting ", Philosophy Compass 2.5 (2007), p. 680-698). Une anatomie bien faite a pour effet, entre autre, de servir la peinture. Cependant, il y aurait bien d'autres justifications pour l'anatomie.

4. La peinture touche un public plus large que l'anatomie. On pense souvent à relier cette distinction à celle du genre littéraire : l'Essai serait, éventuellement, un genre du peintre, car davantage adapté à la philosophie pratique et le Traité un genre de l'anatomiste. Cf. Immerwahr sur cette question.

5. C. Korsgaard, Creating the Kingdom of Ends, Cambridge, Cambridge University Press, 1996 ; et The Sources of Normativity, Cambridge, Cambridge University Press, 1996. Abramson nuance fortement cette interprétation.

6. Mais pas dans le Traité : Hume y est anatomiste. Immerwahr montre que Hume pense qu'il est impossible de faire de l'anatomie et de la peinture dans la même œuvre. Cependant, il lui semble possible de faire les deux dans des œuvres séparées : anatomiste dans le Traité et peintre dans ses Essais moraux et politiques. Abramson pense que Hume parvient à unifier les projets de l'anatomie et de la peinture dans ses Essais moraux et politiques, mais également dans l'Enquête sur l'entendement humain et l'Enquête sur les principes de la morale. 
vue méthodologique, dans sa relation à la théorie du sens moral. Comment comprendre alors cette dernière ? Hume se propose d'examiner le concept pour en extraire les mécanismes cachés. Bien entendu, c'est prendre le risque de mettre à nu les parties les moins aimables et les moins séduisantes de la nature humaine. Or, l'idée est d'affirmer que, derrière la méthode, le projet est également quelque peu différent. La volonté de Hume est moins celle de défendre la cause de la vertu que d'examiner le fonctionnement d'un mécanisme : celui de l'esprit humain.

\section{La cause de la vertu}

Cependant, Hume ne pense pas véritablement desservir la vertu en l'examinant de plus près et en en montrant le fonctionnement, la cause, la logique sous-jacente. Comme nous l'avons suggéré, c'est une question de méthode philosophique, avant tout. Mieux comprendre ce qu'est la vertu, pourquoi elle nous est agréable et parfois même utile, si cela est bien fait, permet aussi de défendre son importance et le rôle qu'elle peut jouer en société. Il explique :

Le même système peut nous aider à former une juste opinion du bonheur aussi bien que de la dignité de la vertu et il peut intéresser tous les principes de notre nature, [aussi bien notre égoïsme et notre orgueil], à embrasser et à chérir cette noble qualité 7 .

Là où Hutcheson aurait tendance à dire qu'il n'est pas nécessaire de comprendre ce qu'est un objet ou comment il fonctionne pour le trouver agréable et l'aimer, Hume part du même principe mais ajoute que pour ce qui est de la vertu, en montrer les principes et révéler qu'il n'y a que du bon à son origine, cela peut aussi servir à prendre sa défense.

Sur ce point, le désaccord entre Hutcheson et Hume reste assez important. Si l'on se réfère à la distinction développée par Mackie, dans son livre Ethics ${ }^{8}$, entre les questions de premier ordre et celles de deuxième ordre, on pourrait affirmer que Hutcheson se soucie fortement des questions de premier ordre. Les questions de premier ordre, ou normatives, indiquent ce que nous devrions faire, quel principe est bon à suivre, quelles actions sont bonnes ou mauvaises (que devrions-nous faire? Quelles vertus

\footnotetext{
7. D. Hume, A Treatise of Human Nature, D. F. Norton \& M. J. Norton (Eds.), Oxford, Oxford University Press, 2007, III iii 6 6. L'extrait est le suivant: The same system may help us to form a just notion of the happiness, as well as of the dignity of virtue, and may interest every principle of our nature [both our selfishness and pride], in the embracing and cherishing that noble quality. (Ma traduction en français). La partie entre crochets, présente dans le manuscrit original, fut retirée lors de son édition en 1739, sans doute à la suite des remarques envoyées par Hutcheson. En effet, Hutcheson renvoie le manuscrit de Hume avec les quelques mots en question, soigneusement rayés.
}

8. Ethics Inventing Right and Wrong, London, Pelican Books, 1977. 
devrions-nous suivre? Qu'est-ce qui est vertueux, qu'est-ce qui est vicieux ?), alors que les questions de second ordre concernent, par exemple, les interrogations sur la nature des valeurs morales, la signification des termes moraux, ou comment nous raisonnons en morale (questions conceptuelles sur la signification de nos jugements moraux, la manière de les analyser, leur logique, par exemple). Pour Hutcheson, la vertu est toujours du côté des sentiments, et non de la raison: on se moque bien, alors, de susciter l'intérêt de l'égoïsme et de l'orgueil, on n'en a pas besoin. Chercher, pour la vertu, une logique, une utilité, une cause et un mécanisme de fonctionnement est dangereux, parce que le fossé qui sépare le raisonnement de l'amour est difficilement franchissable. On ne peut aimer la vertu pour sa logique, son utilité, sa genèse bien pensée, et le but premier de Hutcheson est, justement, de faire aimer la vertu, de montrer que la vertu est naturellement agréable et aimable pour l'homme et qu'elle le serait toujours, même là où elle ne servirait aucune cause et aucun intérêt personnel. Pour quelle raison? Afin qu'elle soit pratiquée. L'enjeu est bel et bien pratique. Or, à partir du moment où la tentative est celle de montrer que la vertu est une création, une élaboration humaine répondant à un but précis, cela ne peut la rendre plus aimable, parce que l'amour ne se décide pas à la suite d'un raisonnement ou du constat d'une utilité quelconque. Pour Hutcheson, cela n'empêche pas que la vertu soit utile et que le raisonnement arrive à cette même conclusion. Cependant, cela ne sert pas sa cause. Le mécanisme et l'élaboration de la vertu ne sont pas de notre ressort, mais semblent s'inscrire dans un système plus grand, dans lequel les éléments fonctionnent en harmonie. L'homme n'a pas besoin de penser pour aimer et la pensée ne rend pas l'objet plus aimable, ni même plus utile, quand l'utilité est déjà là. Mettre en évidence cette utilité fera, au mieux, de la vertu un instrument et il n'est pas certain que cela la serve, elle. Ce sera toute la difficulté, pour Hutcheson, de saisir la distinction de Hume entre vertu artificielle et vertu naturelle : comment un sens de la vertu artificielle peut-il se tourner naturel, à partir du moment où la vertu en question est une construction, un instrument rationnel en vue de servir un but précis ? Plus encore lorsque ce but est associé à l'utilité. En effet, l'utilité peut-elle produire une approbation et un amour? Aime-t-on un instrument? Et de quelle utilité parle-t-on ? Pour qui ?

Que dire alors de la philosophie morale de Hume? Une seule certitude : l'enjeu principal n'est pas celui des questions de premier ordre. Il est même douteux qu'il soit rapporté aux questions de second ordre. Pour Hume, la séparation entre sentiments et raison est aussi effective. Cependant, les distinctions ne sont pas autant marquées. Si certaines vertus sont des instruments en vue de quelque chose et si le but de leur création est quelque chose de bon et de noble, une bonne intention, l'idée de servir des intérêts communs, une communauté entière, alors le but peut être aimable ; et si le but peut être aimable, le moyen, l'instrument pourrait l'être aussi. La vertu est aimable pour ce qu'elle contribue à produire. Cependant, l'approbation d'une action vertueuse ou d'un caractère vertueux ne laisse pas la place à 
une telle réflexion, parce que la réaction humaine est proche de l'instinct, de la perception immédiate et involontaire. Il faut donc comprendre comment une invention humaine, dans le cas d'une vertu artificielle par exemple, ou même comment un mécanisme élaboré où entrent en compte des données de raisonnement comme l'utilité, peut provoquer l'approbation d'un sens naturel, comme par exemple, le sens moral. Toute la question est de saisir, chez Hume, comment la transition devient possible entre le « fabriqué » et le «naturel» et comment on en vient à parler d'un sens moral. En vérité, la philosophie morale de Hume dans le Traité est, avant tout, centrée sur l'explication, sur l'étude de "phénomènes moraux », selon la méthode des sciences empiriques?

\section{Le sens moral}

Qu'est-ce que le sens moral? N'est-ce pas un des ressorts fondamentaux du fonctionnement de la nature humaine, puisqu'il est à l'origine de notre approbation morale ? Le sens moral est ce qui reçoit la perception du caractère vicieux ou vertueux d'une action (de manière apparente ou réelle). Hume écrit, à la section I, 1 de la partie III du Traité :

Il a été observé que rien n'est jamais présent à l'esprit que ses perceptions ; et que toutes les actions de voir, entendre, juger, aimer, haï, et penser, tombent sous cette dénomination. L'esprit ne peut jamais s'exercer dans une quelconque action, que nous ne pouvons comprendre sous le terme de perception; et par conséquent ce terme n'est pas moins applicable à ces jugements, par lesquels nous distinguons le bien moral et le mal, qu'à chacune des autres opérations de l'esprit. Approuver un caractère, en condamner un autre, ne sont que des perceptions différentes ${ }^{10}$.

Si l'approbation morale est le fait d'une perception de l'esprit humain, il n'est pas exclu de parler de sens moral pour désigner une faculté d'avoir

9. La meilleure explication est encore celle de Mackie (Hume's Moral Theory, London, Routledge, 1980, p. 5-6). Il montre, en effet, que Hume, en morale, ne cherche ni à répondre aux questions de premier ordre ni à répondre aux questions de second ordre. Il tente plutôt de suivre le modèle des sciences empiriques: Rather, his question is a demand for an explanation of the sort typically given by the empirical sciences: "Here is this curious phenomenon, human morality, a cluster of attitudes, dispositions, practices, behavioral tendencies, and so on that we find almost universally among men, even in different societies and at different times; why is it there, and how did it develop ?". Hume tente d'examiner un "phénomène moral » comme Newton avait examiné le monde physique.

10. L'extrait est le suivant, Treatise, III i 12 : It has been observ'd, that nothing is ever present to the mind but its perceptions; and that all the actions of seeing, hearing, judging, loving, hating, and thinking, fall under this denomination. The mind can never exert itself in any action, which we may not comprehend under the term of perception; and consequently that term is no less applicable to those judgments, by which we distinguish moral good and evil, than to every other operation of the mind. To approve of one character, to condemn another, are only so many different perceptions. 
une telle sensibilité. Le terme de « sens moral» aurait tendance à positionner Hume, automatiquement, dans le camp des sentimentalistes moraux, à la suite de Shaftesbury et de Hutcheson ${ }^{11}$. Le terme de "sens» et celui de "perception» associés au domaine moral, s'ils sont entendus dans la même logique que celle de Hutcheson, impliquent la considération d'un mécanisme immédiat et involontaire. Involontaire car le fait de percevoir est indépendant de la volonté. Et nous arrivons justement à la considération de l'immédiateté de la perception, trop rapide et primitive pour laisser le temps à l'esprit humain de l'analyser ou de la penser (opérations qu'il ne peut réaliser qu'a posteriori). Pour Hume, le «jugement moral» est une opération de l'esprit et tout ce qui se trouve dans l'esprit est une perception. Les perceptions peuvent être des impressions ou des idées. Hume défend que le «jugement moral» ne relève pas de l'idée. La morale n'est pas la conclusion de la raison: la raison ne peut pas motiver une action ou une affection. La raison est impuissante. La morale est liée au sentiment de plaisir ou de douleur, qui serait davantage du côté d'une impression de réflexion plutôt que d'une idée. De ce fait, si Hume évoque un sens moral, il n'est pas probable qu'il se réfère, dans le Traité, à un "sens commun » ou à un «bon sens» qui le lierait à la raison. Si le «jugement» moral est une impression, même impression de réflexion, cela signifie qu'il ne peut résulter d'un calcul égoïste et être produit en vue d'un intérêt personnel propre car il ne peut être le fruit d'un raisonnement à l'instant même où il est produit. Il ne peut pas être la conclusion d'une déduction rationnelle quelle qu'elle soit. Comme l'explique Hume :

Puisque la morale, par conséquent, a une influence sur les actions et les affections, il s'ensuit qu'elle ne peut être dérivée de la raison; et ce parce que la raison seule, comme nous l'avons déjà prouvé, ne peut jamais avoir une telle influence. La morale excite des passions, et produit ou empêche des actions. La raison d'elle-même est complètement impuissante dans ce cas particulier. Les règles de la moralité, de ce fait, ne sont pas des conclusions de notre raison ${ }^{12}$.

Ainsi, ni Hobbes, ni Locke, ni Mandeville, ni encore Clarke ne sont sur la bonne voie. La motivation est toujours passionnelle et le jugement moral

11. Sur les » sentimentalistes moraux, notamment Shaftesbury, consulter, par exemple, les travaux en français de F. Brugère, L. Jaffro et M. Biziou. En anglais, voir S. Darwall, The British Moralists and the Internal 'Ought' : 1640-1740, Cambridge, Cambridge University Press, 1995. R. Dégremont, "Le Principe de la morale », Revue philosophique de la France et de l'étranger, 1, 135 (2010), p. 45-56. - Pour les rapports de Hume au sentimentalisme moral, on peut consulter l'article de M. Slote et celui de D. Shaw : D. Shaw, «Hume's Moral Sentimentalism», Hume Studies, XIX, 1 (April, 1993), p. 31-54 et M. Slote, "Moral Sentimentalism », Ethical Theory and Moral Practice, 7, 1 (Mar., 2004), p. 3-14.

12. Hume, op. cit., III i 16 : Since morals, therefore, have an influence on the actions and affections, it follows, that they cannot be deriv'd from reason; and that because reason alone, as we have already prov'd, can never have any such influence. Morals excite passions, and produce or prevent actions. Reason of itself is utterly impotent in this particular. The rules of morality, therefore, are not conclusions of our reason. 
est lié aux sentiments. Ce constat est déjà celui de Francis Hutcheson, quelques années auparavant. Il écrit, par exemple dans son Essai :

Le raisonnement ou l'intellect ne semble provoquer aucune nouvelle espèce d'idées, mais découvrir ou discerner les relations existant avec celles qui sont déjà reçues. La raison montre quels actes sont en conformité avec une loi, une volonté supérieure, ou quels actes tendent vers le bien privé ou vers le bien public. De la même façon, la raison découvre des tendances contraires d'actions contraires. [...] Mais quand nous approuvons une action généreuse et bénéfique, considérons si ce sentiment, ou cette action, ou modification de l'âme, ressemble davantage à un acte de contemplation, tel que celui-ci, «quand des lignes droites se coupent, les angles opposés par le sommet sont égaux », ou bien cette inclination que nous avons pour une belle forme, une composition harmonieuse, un son agréable ${ }^{13}$.

Pour Hutcheson, l'approbation morale est le fait d'une sensibilité particulière, une perception du caractère vicieux ou vertueux d'une action dans la perception même de l'action. Une perception dans les perceptions. Nous avons, en effet, cinq sens externes et les perceptions qui leur correspondent. L'œil voit, l'oreille entend, etc. Hutcheson fait une analogie entre les sens externes et deux sens internes : le sens moral et le sens de la beauté. Le sens moral est le récepteur d'une perception interne, c'est-à-dire la capacité que nous avons à réagir envers une sensibilité particulière. De même, le sens de la beauté est le récepteur d'une perception interne. Il existe une première analogie de fonctionnement entre le sens interne moral et le sens interne de la beauté. Il existe une seconde analogie de fonctionnement entre les sens internes, d'une part, et les sens externes, d'autre part. Prenons pour exemple le sens moral. Il est sensible à une perception interne et cette perception interne se forme à partir des données reçues dans les perceptions des sens externes : je vois une certaine action, un sens externe entre ainsi en jeu. La perception externe de cette action, autrement dit, la vision, me donne une information particulière qui produit immédiatement et involontairement en moi l'idée de vertu accompagnée d'un plaisir : perception interne du sens moral. Cette perception du sens moral naît dans la perception externe même de l'action (au moment où je vois). Bien entendu, ce n'est pas n'importe

13. F. Hutcheson, Essai sur la nature et la conduite des passions et affections avec Illustrations sur le sens moral, trad. O. Abiteboul, Paris, L'Harmattan, 2003, I, p. 47. F. Hutcheson, An Essay on the Nature and Conduct of the Passions and Affections. With Illustrations upon the Moral Sense. By Francis Hutcheson, LL.D. Late..., Glasgow, Robert and Andrew Foulis editors, 1769 ( $3^{\mathrm{e}}$ edition - $1^{\text {ère }}$ édition : 1728), I, p. 215-216. L'extrait est le suivant : Reasoning or intellect seems to raise no new species of ideas, but to discover or discern the relations of those received. Reason shews what acts are conformable to a law, a will of a superior; or what acts tend to private good, or to public good: in like manners, reason discovers contrary tendencies of contrary actions. [...] But when we approve a kind beneficent actions, let us consider whether this feeling, or action, or modification of the soul more resembles an act of contemplation, such as this when straight lines intersect each other, the vertical angles are equal; or that liking we have to a beautiful form, an harmonious composition, a grateful sound. 
quelle action qui peut susciter en moi l'idée de vertu : ce sont des qualités particulières perçues dans l'action qui provoquent cela. Le principe d'un sens interne fonctionne un peu comme le modèle de l'oreille musicale, où une personne entend les sons et perçoit en même temps l'harmonie qui s'en dégage ou non. En plus des sons perçus, il y a cette perception de l'harmonie. La différence est que tout le monde possède un sens moral, naturellement. Sur ce plan, les hommes sont totalement égaux de naissance. De même, l'analogie se fait avec le sens de la beauté, car Hutcheson considère que l'homme possède une sensibilité particulière qui lui fait percevoir une certaine qualité dans l'objet, qualité qui produit instantanément l'idée de la beauté. Une perception interne à partir des perceptions externes, une nouvelle fois.

La définition du sens moral de Hutcheson reste celle d'une sensibilité qui laisse supposer une faculté. Quant à la faculté même, nul ne sait ce qu'elle est, exactement. Hutcheson ne cherche pas à affirmer que nous possédons un organe sensoriel spécifique à la sensibilité morale. Il montre simplement que la raison à elle seule ne peut être la source de notre approbation ou condamnation morale et que la vertu est une idée accompagnée d'un plaisir particulier et le vice une idée accompagnée d'une douleur particulière. Or, cette idée plaisante n'est pas le fruit d'une réflexion, mais se forme dans la perception même de certaines qualités des actions observées, certaines qualités perçues chez certains caractères ou dans certaines actions. L'effet qui montre que l'approbation morale fonctionne comme une perception laisse supposer la cause : un sens moral. Hutcheson montre donc que l'important n'est pas tant de décrire ce qu'est le sens moral que de montrer ce qu'il fait et ce qu'il implique pour la nature humaine.

Et de fait, Hume choisit de commencer sa troisième partie du Traité par l'affirmation de l'existence d'un sens moral. Il écrit :

Les distinctions morales dérivent d'un sens moral $^{14}$.

14. Treatise, III i 2 : Moral distinctions deriv'd from a moral sense. La présence du sens moral chez Hume a suscité de nombreux débats, notamment entre les défenseurs d'une vision projectionniste et ceux d'une vision réaliste de sa philosophie. Les projectionnistes pensent que les critères moraux ne sont pas réellement ceux des agents ou des actions elles-mêmes. Ils pensent, au contraire, que la philosophie morale de Hume est subjectiviste : les propriétés morales sont dans l'esprit plutôt que dans l'objet. Les réalistes, eux, soulignent l'importance du sens moral et le lien avec Hutcheson pour défendre que ce sens nous permet de prendre connaissance, à travers les sentiments, des qualités de vertu ou de vice des objets euxmêmes. Sur la comparaison entre les deux positions, voir A. E. Pitson mais aussi D. Shaw (cf. Bibliographie, p. 60). A. E. Pitson, à l'occasion, montre le rapprochement à effectuer entre le sens moral de Hume et les sens externes. Les deux peuvent nécessiter une correction vis-à-vis des circonstances dans lesquels ils se trouvent. Les sens externes doivent distinguer les apparences des objets eux-mêmes, le sens moral doit distinguer les actions telles qu'elles nous apparaissent et leurs causes mentales (intentions). A. E. Pitson montre, au final, que le sens moral implique la sensibilité envers à la fois une intention et une action : des causes et des effets. $\mathrm{Ni}$ les projectionnistes ni les réalistes ne lui semblent donner une explication satisfaisante de Hume. Shaw propose de faire un parallèle avec la théorie des qualités 
Cependant, il n'a pas exactement la même idée que Hutcheson lorsqu'il traite de cette question. Hutcheson avait écrit, en effet, dans sa Recherche :

Cette détermination naturelle à approuver et à admirer, ou à haïr et à honnir les actions, est sans doute une qualité occulte ${ }^{15}$.

Pour Hume, le sens moral ne peut pas être une «qualité occulte». L'anatomiste doit mettre à jour les parties cachées de la nature humaine et expliquer comment elles fonctionnent. De ce fait, Hume entame la dissection du sens moral jusqu'à en révéler ce qui, pour lui, en est l'ossature. Et contre toute attente, l'opération débouche sur un autre concept : celui de sympathie.

\section{La sympathie}

$\mathrm{Au}$ sujet du lien entre sens moral et sympathie, Hume donne l'explication suivante :

Peu de connaissance des sujets humains est requise pour s'apercevoir que le sens de la moralité est un principe inhérent dans l'âme, et un des plus puissants qui entrent dans sa composition. Mais ce sens doit certainement gagner une force nouvelle quand, réfléchissant sur lui-même, il approuve les principes desquels il dérive, et ne trouve rien à sa naissance et à son origine qui ne soit grand et bon. Ceux qui réduisent le sens de la moralité à des instincts originaux de l'esprit humain peuvent défendre la cause de la vertu avec une autorité suffisante; mais il leur manque l'avantage de ceux qui expliquent ce sens par une sympathie étendue à l'humanité ${ }^{16}$.

Il faut bien faire attention, ici, à ne pas confondre la sympathie et le sens moral : les deux ne sont pas la même chose et l'idée n'est pas de remplacer

secondes de Locke, en tant que pouvoirs des objets de provoquer certaines perceptions dans l'esprit de l'observateur, afin de mieux comprendre ce que sont les qualités de vice ou de vertu. De là, il constitue une théorie intermédiaire qu'il nomme combined autobiographical/ powers version: la vertu est le pouvoir d'une action de susciter en moi une approbation et de la susciter chez n'importe quel observateur d'un point de vue général et impartial. Ce pouvoir vient des faits eux-mêmes, de leur utilité et du fonctionnement de la nature humaine à travers la sympathie, principalement.

15. F. Hutcheson, Recherche sur l'origine de nos idées de la beauté et de la vertu, Balmès (trad.), Paris, Vrin, 1991, II, 7, p. 238. - F. Hutcheson, An Inquiry into the Original of our Ideas of Beauty and Virtue in Two Treatises, Glasgow, Robert and Andrew Foulis editors, 1772, II, 7, p. 247. L'extrait est le suivant: This natural determination to aprove and admire, or hate and dislike actions, is, no doubt, an occult quality.

16. Hume, Treatise, III iii 63 : It requires but very little knowledge of human affairs to perceive, that a sense of morals is a principle inherent in the soul, and one of the most powerful that enters into the composition. But this sense must certainly acquire new force, when reflecting on itself, it approves of those principles, from whence it is deriv'd, and finds nothing but what is great and good in its rise and origin. Those who resolve the sense of morals into original instincts of the human mind, may defend the cause of virtue with sufficient authority, but want the advantage, which those possess, who account for that sense by an extensive sympathy with mankind. 
le sens moral par un nouveau concept de sympathie, un concept qui jouerait le rôle de «super sens moral». Pour Hume, la sympathie est l'origine de la formation du sens moral. Elle est composée des principes qui formeront le sens moral. Entre la sympathie et le sens moral, il y a une transition : le passage de l'un à l'autre n'est pas immédiat. Voyons comment cela fonctionne.

La véritable cause de l'action morale est la passion, c'est-à dire une impression seconde ou réflexive. C'est de cette passion que naissent l'approbation ou la désapprobation. Or, la recherche de l'origine de cette passion nous apprend ce qu'est la sympathie : un principe de communication des passions d'une personne à une autre. La sympathie permet de ressentir du plaisir à partir des sentiments d'autrui, et permet, ainsi, la formation de nombreuses passions comme l'orgueil, l'humilité, l'amour ou la haine. Le sens de la beauté, également, en dépend, pour une grande part. Elle est à l'origine des caractères nationaux, mais c'est surtout en tant que principe du sens moral qu'elle doit retenir notre attention, ici.

Le sens moral dépend, en effet, d'une extension de la sympathie par les règles générales. La sympathie est corrigée par des règles générales parce que l'homme est partial : or, le sens moral doit s'étendre au-delà des préférences. Les hommes apprennent à neutraliser la pure subjectivité de leurs jugements, à écarter l'intérêt personnel pour gagner de la distance et réagir impartialement. Hume observe, d'ailleurs, que les corrections des apparences momentanées des choses se font régulièrement, par tous nos sens.

La sympathie s'explique de cette manière : au commencement, nous avons une impression de nous-mêmes. Cette impression est première et donc, toujours très vive. Une partie de cette vivacité est transférée aux objets auxquels nous sommes liés par sympathie. Avec autrui, nous sommes liés par les relations de contiguïté et ressemblance : les esprits de tous les hommes sont semblables par leurs sentiments et leurs opérations, c'est pour cette raison que Hume considère qu'aucun être humain ne peut ressentir une affection dont tous les autres seraient incapables, à quelque degré que ce soit. Les actions d'autrui sont, également, interprétées selon nous, comme des signes des passions qui les animent, ce qui ajoute une relation de causalité entre les actions des autres, leurs passions que nous croyons interpréter selon des signes qui nous «parlent». Le rôle de l'imagination est fondamental dans le processus de la sympathie: on se réjouit ou l'on souffre parce que l'on imagine l'émotion d'autrui à travers des indices. Tout ce système de relations conduit l'impression de nous-mêmes à l'idée des sentiments ou passions d'autrui et nous les font concevoir d'une façon très vive. Les idées de soi et des autres sont toujours associées par la sympathie $^{17}$.

17. Hume explique le mécanisme de la sympathie de la façon suivante, Treatise, III iii 17 : When I see the effects of passion in the voice and gesture of any person, my mind immediately passes from these effects to their causes, and form such a lively idea of the passion, as is 
Le sens moral est le fruit d'une sympathie étendue à l'humanité, une sympathie dépourvue de considérations propres, une sympathie « impartiale». Tous les hommes sont capables d'éprouver une telle sympathie, même si certains facteurs jouent toujours : celui qui a beaucoup souffert du froid est plus sensible aux manifestations qui y sont liées. En même temps, aucun être humain n'est assez éloigné d'un autre être humain au point d'être toujours totalement insensible à un certain affect ou à une certaine passion : les différences entre les hommes ne sont pas si grandes. De même, le fait de partager avec autrui une émotion, c'est-à dire de se sentir triste quand l'autre est triste par exemple, nous fait nous intéresser à une autre personne que nous-mêmes : il s'agit d'une extension de notre propre intérêt jusqu'à celui des autres ${ }^{18}$. Maintenant, comment une telle sympathie étendue à l'humanité devient-elle un sens moral?

Elle le devient parce que le mécanisme de la sympathie est caché. Nous n'avons pas conscience, selon Hume, du fonctionnement même de notre sens moral. Et ce, parce que dans l'expérience, l'approbation ou la condamnation morale sont instantanées. Elles sont si rapides, la communication entre passions et sentiments est si vive et le plaisir ou la peine si immédiats que cela ne laisse pas le temps d'analyser ou de réfléchir. L'expérience a la vitesse et la force d'une perception, au point que, ce qui est un mécanisme de communication devient l'équivalent d'une sensibilité particulière aux passions et sentiments des autres. Notre interprétation des signes est automatique. Cet automatisme vient, sans aucun doute, de l'expérience ; de ce que Hume nomme indifféremment « habitude» ou « coutume ». La seule chose qui peut permettre à Hume de parler de sens moral à la place de son concept de sympathie étendue à l'humanité entière est la force de l'habitude. En s'habituant à interpréter les signes, à observer les caractères et les actions avec attention, en s'habituant à prendre de la distance vis-à-vis de notre partialité, de notre intérêt personnel, en s'habituant à corriger notre vision des choses pour essayer de prendre en considération un point de vue général, celui de l'humanité entière, on parvient à rendre naturel ce qui ne l'était pas forcément et à se constituer un sens moral, dont l'origine et les principes n'altèrent pas ce qu'il est. Le résultat est bien un sens moral, même si Hume révèle qu'il dérive d'autres principes. Le sens est naturel lorsqu'il ne résulte plus d'aucun effort mais que l'habitude en a fait une seconde nature: une sensibilité naturelle issue d'un mécanisme complexe. Dans la pratique, notre sympathie finit donc par échapper à notre contrôle, elle devient involontaire et immédiate.

presently converted into the passion itself. La traduction serait la suivante : «Quand je vois les effets d'une passion dans la voix et la gestuelle d'une personne, mon esprit passe immédiatement de ces effets à leurs causes, et forme une idée tellement vive de la passion, qu'elle se convertit en la passion elle-même ».

18. Sur ce point, consulter M. Biziou, « Kant et Smith, critiques de la philosophie morale de Hume », Revue Philosophique de la France et de l'Étranger, 190, 4, (Oct.-Déc. 2000), p. 449-464. Agir de manière désintéressée, par exemple, c'est inclure l'intérêt des autres comme le sien propre. Le désintéressement et l'impartialité sont la clé de la sympathie. 
Il est, maintenant, intéressant d'observer que les effets du sens moral chez Hutcheson et de la sympathie étendue à l'humanité entière et renforcée par l'habitude chez Hume doivent avoir, au final, les mêmes effets. Les deux nous intéressent au bien de la société, les deux nous rendent agréables certaines fins qui ne nous sont pas directement liées, les deux nous rendent sensibles au bonheur des autres hommes. Enfin, bien que les deux concernent tous les êtres humains, car chacun en a la faculté, les deux ne constituent pas, à proprement parler, un motif d'action suffisant, à eux seuls. Dans les deux cas, les évaluations morales procurent un plaisir ou une souffrance particuliers : à caractère public, tourné vers les autres. On part de l'individu, de sa sensibilité, pour penser les relations aux autres, à un groupe, à une communauté. En revanche, on l'aura compris, Hume ne fait pas de la sympathie, et encore moins du sens moral, le tout de l'expérience morale, puisque d'autres principes entrent en considération, notamment dans l'élaboration d'une vertu artificielle. Cependant, ce n'est pas parce que le sens moral joue un rôle plus faible dans la philosophie morale du Traité de Hume que dans la philosophie de Hutcheson, qu'il n'en est pas, pour autant, un des principes fondamentaux.

\section{Conclusion}

Une des choses que met en évidence la comparaison de l'anatomiste et du peintre, c'est que les deux méthodes sont deux méthodes d'analyse du même objet: il s'agit toujours d' « examiner l'esprit», selon les propres mots de Hume. Que ce soit dans les œuvres de Hutcheson ou dans celles de Hume, l'idée est bien d'étudier la nature humaine et son fonctionnement. Cependant, pour Hutcheson, l'accent est mis sur l'action, la mise en pratique, les effets du fonctionnement. Lorsque Hutcheson étudie la nature humaine, il a toujours en vue les implications pratiques d'une telle étude, c'est pour cette raison que son œuvre est fondamentalement éthique et politique. Il fait de la philosophie pour éduquer et donner les moyens à ses étudiants et lecteurs d'être de bons citoyens. Chez Hume, il n'y a pas la même préoccupation, du moins pas au même degré. Hume considère qu'un philosophe comme Hutcheson n'est pas allé assez loin dans la découverte des principes qui régissent le fonctionnement de l'esprit humain. Son travail philosophique dans le Traité révèle moins un souci d'action qu'un souci d'étude et d'analyse d'un objet scientifique. La vertu est l'objet d'une étude visant à développer la connaissance de ce qu'est l'objet et de comment il fonctionne. Contrairement à Hutcheson, Hume ne fait pas de politique lorsqu'il traite du sens moral et de la vertu. Il se concentre sur les principes, les causes ${ }^{19}$. 
Pourtant, il est intéressant de voir que Hume ne se dissocie pas totalement du projet philosophique de Hutcheson parce que son idée est davantage celle de compléter les parties manquantes, les lacunes de ce dernier. Que ce soit avec Hutcheson, ou avec un autre philosophe tel que Mandeville ou Shaftesbury, l'idée n'est pas tant celle de "corriger » ou de « rectifier» que celle d'aller plus loin ${ }^{20}$. Ce n'est pas que Hume est véritablement réticent à l'idée de défendre la cause de la vertu, mais c'est plutôt que Hutcheson l'a déjà fait. Quand Hume aborde la question du sens moral et de la vertu, il se range déjà, d'une certaine manière, du côté des sentimentalistes moraux : il ne va donc pas répéter ce qui a été dit. Affirmer qu'il existe dans le monde de la bienveillance comme motif d'action, même si elle est très faible et insuffisante, suffit à remettre en cause le système philosophique d'un Hobbes ou d'un Mandeville et suffit donc à remettre en cause l'usage politique que certaines institutions, notamment religieuses, peuvent faire d'une philosophie qui place l'intérêt personnel comme motif unique des actions humaines. Hume n'a pas besoin d'en dire plus. Cependant, Hume est aussi un héritier naturel de certains aspects de la philosophie de Hobbes et de Mandeville. L’idée, pour Hume, est de dépasser les points de désaccord entre défenseurs du sens moral et défenseurs de l'intérêt particulier, afin de voir si cela peut servir l'élaboration et la poursuite d'une véritable science de la nature humaine.

uniquement descriptive ou explicative. Il défend que cette distinction, entre normatif et explicatif, n'est pas la distinction que Hume fait lorsqu'il se place du point de vue de l'anatomiste. Hume ne dit pas qu'il s'oppose aux jugements normatifs, il en fait lui-même. Shaver dit plutôt que Hume ne veut pas «montrer explicitement pourquoi la moralité devrait nous engager ». Shaver considère également que la philosophie morale de Hume est parfois justificative. D'une certaine manière, Hume s'occupe indirectement de philosophie pratique dans le Traité. En vérité, notre propos n'a pas pour objectif d'aller contre ce que dit Shaver. Il n'est pas question, pour nous, d'affirmer, en matière morale, que Hume ne s'occupe jamais de questions de premier ordre, ni même de second ordre. Nous n'essayons pas non plus de défendre que Hutcheson ne fait pas de philosophie explicative ou descriptive. Il est plutôt question de montrer, conformément à ce qu'affirme Mackie, que la tendance principale et l'objectif premier de Hume ne sont pas, dans le Traité, de faire une philosophie morale normative. Il privilégie fortement une philosophie explicative, dans la lignée de son projet; or, la figure de l'anatomiste illustre bien une telle démarche (même si elle n'illustre pas que cela). Dans le cadre d'une comparaison entre nos deux philosophes, il est encore plus marquant que la préoccupation pratique, au sens de guider les hommes dans leurs actions, est davantage le souci de Hutcheson que de Hume.

20. Treatise, introduction, $\S 7$ : the space of time is nearly equal to that betwixt my Lord Bacon and some late philosophers [note: Mr. Locke, my Lord Shaftesbury, Dr. Mandeville, Mr. Hutcheson, Dr. Butler, \&c.] in England who have begun to put the science of man on a new footing, and have engaged the attention, and excited the curiosity of the public. - Traduction : «L'espace temps est presque égal à celui entre Lord Bacon et ces quelques derniers philosophes [note: Locke, Shaftesbury, Mandeville, Hutcheson, Butler, \&c.] en Angleterre qui ont commencé à placer la science de l'homme sur une nouvelle base, ont retenu l'attention et excité la curiosité du public ». - L'important, ici, est le mot begun, « commencé ». Hume se présente comme l'héritier naturel de ceux qui ont commencé à élaborer une nouvelle base pour la science de l'homme. 
L'anatomiste du Traité ne détruit pas le travail du peintre. Au contraire, il l'aide, parfois, à être plus précis dans ses réalisations. Il peut apporter de nouveaux éléments et étudier le même objet d'un autre point de vue. L'anatomiste et le peintre peuvent travailler ensemble parce que les découvertes de l'un peuvent toujours faire avancer l'autre. Les points de vue ne sont pas forcément conciliables, mais ils n'en sont pas moins tous deux indispensables à l'élaboration et au développement de la philosophie morale. C'est l'idée de Hume dans le Traité et c'est de cette manière qu'il aborde les concepts clés de la philosophie de Hutcheson. Hume se place du côté de la science et laisse l'art à son contemporain. La crainte de Hutcheson sera toujours celle de laisser échapper l'élément fondamental de l'objet : à y regarder de si près, le risque est de se rendre aveugle. Pour ce qui est du sens moral, il serait inexact d'affirmer qu'il n'est pas un élément important de la philosophie du Traité de Hume. Et ce, parce que Hume part du concept de Hutcheson pour élaborer la partie de son œuvre sur la morale. En vérité, il part du résultat. Le constat général de l'observation de la nature humaine est que nous avons un sens moral. Ce sens moral est un principe d'approbation naturel. Cependant, si on s'interroge sur l'origine d'un tel sens, on verra qu'il est issu d'un mécanisme complexe, un mécanisme de sympathie. Peuton alors encore parler de sens moral à la place de sympathie ? Oui. Tout dépend du point de vue autour duquel on se place. Si l'on considère le mécanisme caché, mécanisme dont nous n'avons pas même conscience, au moment de l'action, dans la vie de tous les jours, on parlera de sympathie. $\mathrm{Si}$, par contre, on décide de parler du résultat, de la perception que nous avons au moment de l'action, dans la vie pratique, alors on parlera de sens moral, parce que c'est sous cette forme que l'expérience morale se manifeste à nous. On pourra alors dire, comme Hume :

De cette façon, quand nous déclarons qu'une action ou un caractère sont vicieux, tout ce que nous voulons dire est que, selon la constitution de notre nature, nous expérimentons une sensation ou sentiment de censure quand nous les contemplons. Le vice et la vertu, par conséquent, peuvent être comparés aux sons, couleurs, chaleur et froid, lesquels, selon la philosophie moderne, ne sont pas des qualités dans les objets, mais des perceptions dans l'esprit. Et cette découverte de la morale, comme celle de la physique, doit être vue comme un progrès considérable dans les sciences spéculatives, bien que, exactement comme celle-ci, elle a peu ou n'a aucune influence dans la pratique ${ }^{21}$.

Et nous conclurons, une nouvelle fois, sur la différence de point de vue entre la philosophie de Hutcheson et celle de Hume : là où Hume affirme

\footnotetext{
21. Op. cit., III i 126 : So that when you pronounce any action or character to be vicious, you mean nothing, but that from the constitution of your nature you have a feeling or sentiment of blame from the contemplation of it. Vice and virtue, therefore, may be compar'd to sounds, colours, heat and cold, which, according to modern philosophy, are not qualities in objects, but perceptions in the mind: and this discovery in morals, like that other in physics, is to be regarded as a considerable advancement of the speculative sciences; tho', like that too, it has little or no influence on practice.
} 
tout naturellement le peu d'impact de la présente découverte, Hutcheson s'appliquera, toute sa vie, à démontrer comment une telle découverte a tout à voir avec la pratique et a donc, bien au contraire, une influence à ne surtout pas négliger; et ce, dans toutes ses implications. Or, il s'agit là d'une différence de point de vue, répéterons-nous; de point de vue ? Une différence qui, dans tous les cas, est bien révélatrice de la relation délicate et complexe qui est celle d'un peintre et d'un anatomiste, en admettant, par ailleurs, que Hutcheson ait un jour accepté, de bonne grâce, la comparaison faite par Hume. 


\section{Bibliographie}

\section{Euvres de Hutcheson et de Hume}

Hume, D., A Treatise of Human Nature, D. F. Norton \& M. J. Norton (Eds.), Oxford, Oxford University Press, 2007.

Hutcheson, F., An Essay on the Nature and Conduct of the Passions and Affections. With Illustrations upon the Moral Sense. By Francis Hutcheson, LL.D. Late..., Glasgow, Robert and Andrew Foulis editors, 1769.

Hutcheson, F., An Inquiry into the Original of our Ideas of Beauty and Virtue in Two Treatises, Glasgow, Robert and Andrew Foulis editors, 1772. Hutcheson, F., Collected works of Francis Hutcheson, Hildesheim, Zürich, New York, G. Olms, 1990.

Hutcheson, F., Essai sur la nature et la conduite des passions et affections avec illustrations sur le sens moral, trad. fr. par Olivier Abiteboul, Paris, L'Harmattan, 2003.

Hutcheson, F., Recherche sur l'origine de nos idées de la beauté et de la vertu, trad. fr. par Anne-Dominique Balmès, Paris, Vrin, 1991.

\section{Littérature secondaire et philosophie contemporaine}

Abramson, K., "Hume's Distinction between Philosophical Anatomy and Painting ", Philosophy Compass 2.5 (2007), p. 680-698.

Biziou, M., Shaftesbury. Le sens moral, Paris, PUF, 2005.

Abramson, K, «Kant et Smith, critiques de la philosophie morale de Hume », Revue Philosophique de la France et de l'Etranger, 190, 4, (Octobre-Décembre 2000), p. 449-464.

Brahami, F., «Sympathie et individualité dans la philosophie politique de David Hume », Revue Philosophique de la France et de l'Étranger, 182, 2 (avril-juin 1992), p. 201-227.

Brugère, F., Théorie de l'art et philosophie de la sociabilité selon Shaftesbury, Paris, Champion, 1999.

Buckle, S., Hume's Enlightenment Tract, Oxford, Oxford University Press, 2001. 
Cunningham, A. S., "The Strength of Hume's "Weak" Sympathy », Hume Studies 30, 2 (2004), p. 237-256.

Darwall, S., The British Moralists and the Internal 'Ought' : 1640-1740, Cambridge, Cambridge University Press, 1995. R. Dégremont, «Le Principe de la morale », Revue philosophique de la France et de l'étranger 1, 135 (2010), p. 45-56.

Gill, M. B., "Fantastick Associations and Addictive General Rules: A Fundamental Difference between Hutcheson and Hume», Hume Studies XXII, 1 (April, 1996), p. 23-48.

Immerwahr, J., «The Anatomist and the Painter: The Continuity of Hume's Treatise and Essays », Hume Studies XVII, 1 (April, 1991), p. 1-14.

Jaffro, L. (dir.), Le sens moral. Une histoire de la philosophie morale de Locke à Kant, Paris, PUF, 2000.

Jaffro, L., Éthique de la communication et art d'écrire. Shaftesbury et les Lumières anglaises, Paris, PUF, 1998.

Kirby, B., Hume, "Sympathy, and the Theater », Hume Studies XXVIX, 2 (November, 2003), p. 305-325.

Korsgaard, C., Creating the Kingdom of Ends, Cambridge, Cambridge University Press, 1996.

Korsgaard, C., The Sources of Normativity, Cambridge, Cambridge University Press, 1996.

Lazzeri, C., «La Querelle de l'intérêt et de la sympathie. Petite anthologie philosophique des $\mathrm{XVII}^{\mathrm{e}}$ et $\mathrm{XVIII}^{\mathrm{e}}$ siècles », Revue du MAUSS 1, 31 (2008), p. 33-66.

Mackie, J. L., Hume's Moral Theory, London, Routledge, 1980.

Mackie, J. L., Ethics Inventing Right and Wrong, Londres, Pelican Books, 1977.

Malherbe, M., "Hume and the Art of Dialogue », in Hume and Hume's Connections, M. A. Stewart (dir.), J. P. Wright (dir.), University Park, Pennsylvania State University Press, 1995, p. 201-223.

Moore, J., "Hume and Hutcheson », in Hume and Hume's Connections, M. A. Stewart (dir.), J. P. Wright (dir.), University Park, Pennsylvania State University Press, 1995, p. 23-57.

Norton, D. F., David Hume, Common-Sense Moralist, Sceptical Metaphysician, Princeton, Princeton University Press, 1982.

Pitson, A. E., " Projectionism, Realism, and Hume's Moral Sense Theory », Hume Studies XV, 1 (April 1989), p. 61-92.

Pitson, T., «Sympathy and Other Selves», Hume Studies XXII, 2 (November, 1996), p. 255-272.

Scott, W. R., Francis Hutcheson: His Life, Teaching and Position in the History of Philosophy, Londres, Thoemmes Press, 1900/1992. 
Shaver, R., «Hume's Moral Theory? », History of Philosophy Quarterly, 12, 3 (Jul., 1995), p. 317-331.

Shaw, D., « Hume's Moral Sentimentalism », Hume Studies XIX, 1 (April, 1993), p. 31-54.

Slote, M., "Moral Sentimentalism », Ethical Theory and Moral Practice, 7, 1 (Mar., 2004), p. 3-14.

Smith, N. K., The Philosophy of David Hume: A Critical Study of its Origins and Central Doctrines, Londres et New York, Macmillan, 1941.

Stewart, M. A., «Two Species of Philosophy: The Historical Significance of the First Enquiry », in Reading Hume on Understanding, Peter Millican (dir.), Oxford, Oxford University Press, 2002, p. 67-95.

Volpato Dutra, D. J., "Hume contra Hume», Revista Portuguesa de Filosofia 60, 1 (Jan.-Mar., 2004), p. 81-107.

Wood, P. B., «Science and the Pursuit of Virtue in the Aberdeen Enlightenment », in Studies in the Philosophy of the Scottish Enlightenment, M. A. Stewart (dir.), Oxford, Clarendon Press, 1990, p. $127-49$. 